\title{
Ergiebige Gedankenarbeit auf schwierigem Terrain
}

\section{Bruno Kesseli}

Dr. med. et lic. phil., Chefredaktor (Text und Bilder)

Der Informations- und Gedankenaustausch, zu dem das Schweizerische Institut für ärztliche Weiter- und Fortbildung SIWF und das Kollegium der Dekane der schweizerischen medizinischen Fakultäten traditionellerweise im Januar einladen, brachte auch in der jüngsten Auflage spannende Erkenntnisse, Anregungen und Impulse rund um die ärztliche Aus- und Weiterbildung.

«Der Krug geht zum Brunnen, bis er bricht», weiss das Sprichwort. Nicht zum Brunnen, sondern nach Brunnen gehen seit Jahren die Teilnehmerinnen und Teilnehmer der «Journée de réfléxion». Mit dieser Tradition zu brechen, scheint vorderhand kein Thema zu sein - auch die Tagung vom Januar dieses Jahres bot dafür keinen Anlass. Denn die gastgebenden Präsidenten Werner Bauer (SIWF) und Klaus Grätz (Collège des doyens) hatten es verstanden, einmal mehr ein Programm zusammenzustellen, das die Diskussion um Fragen der ärztlichen Weiterbildung belebte und Anstösse zu deren Weiterentwicklung gab.
Kennzeichnend für die Veranstaltung war eine wohltemperierte Mischung aus (Impuls-)Referaten und zeitlich grosszügig anberaumten Blöcken, die dem direkten Austausch unter den Tagungsteilnehmerinnen und -teilnehmern dienten. Dabei handelte es sich fast ausnahmslos um Expertinnen und Experten für die ärztliche Bildung, die sehr unterschiedliche Perspektiven einbrachten, was der Diskussion zugute kam, aber auch Spannungsfelder zutage treten liess. Für einmal aus Termingründen nicht vertreten war das Bundesamt für Gesundheit. Dies war insofern bedauerlich, als die Meinung von BAG-Vertretern zu der Medizinischen

Fakultäten der Schweiz

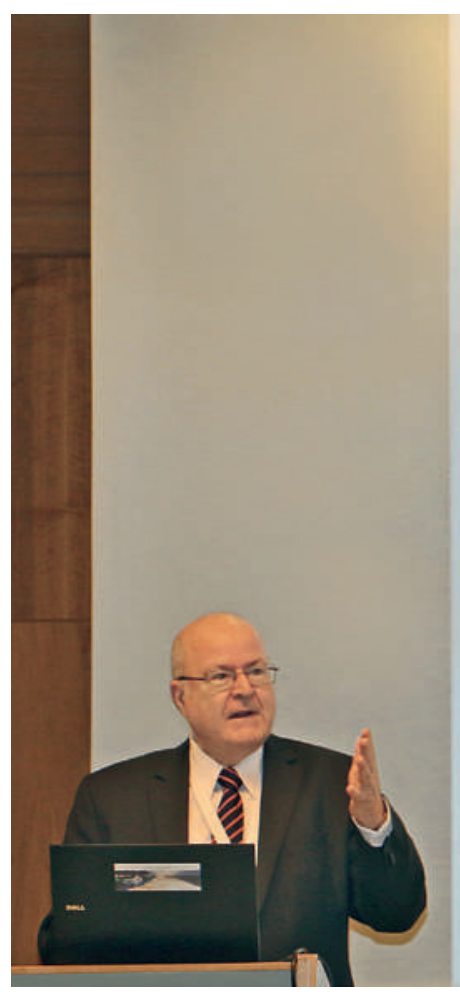

\section{Journée de réflexion 2015}




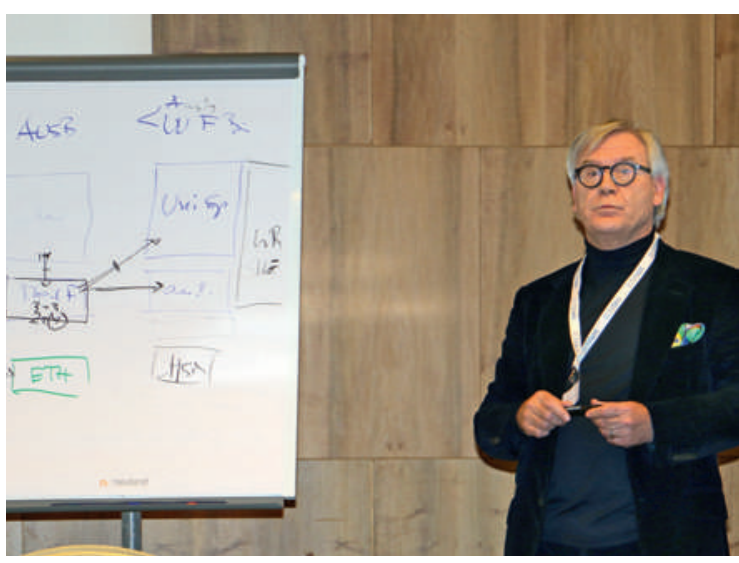

Schnittstellen: Klaus Gräz bei der Moderation des ersten Themenblocks.

verschiedenen Fragen speziell interessiert hätte und diese wohl ihrerseits mit einigen wertvollen Anregungen im Gepäck nach Bern zurückgereist wären.

\section{Bessere Vernetzung von Universitäts- spitälern, medizinischen Fakultäten und weiteren Spitälern}

Zentrales Thema des ersten Halbtags waren vor dem Hintergrund des Wandels der medizinischen Versorgungsstrukturen die Schnittstellen zwischen den Universitätsspitälern, den medizinischen Fakultäten und den weiteren Spitälern im Bereich der ärztlichen Aus- und Weiterbildung. Klaus Grätz, der diesen Themenblock moderierte, betonte, dass Handlungsbedarf bezüglich der Zusammenarbeit von Universitätsspitälern und medizinischen Fakultäten bestehe. Das Problem sei indes erkannt und Verbesserungen seien eingeleitet, etwa mit der Bildung des «Club des Quinze» und des Verbands Universitätsmedizin Schweiz, die ein "engeres Zusammenrücken» dieser Institutionen gewährleisten sollten.

Verschiedene Votanten, darunter Iris Baumgartner, Vizedekanin Weiterbildung der Medizinischen Fakultät in Bern, konstatierten Defizite bezüglich der Weiterbildung an Universitätsspitälern im Vergleich zu peripheren Spitälern, "wo der Weiterbildner etwas gilt». Eine Aufwertung wäre dringlich, auch angesichts der Tatsache, dass die Universitätsspitäler 35 Prozent der Weiterbildungsstellen stellten, doch fehlten dafür die «Incentives». Es war das erste Mal, dass dieser Begriff fiel, der sich im Verlauf der Tagung zu einer Art «Zauberwort» entwickelte.

Ihr Kollege Peter Eggli, Dekan der Medizinischen Fakultät in Bern, bedauerte, dass von der Fakultät aus

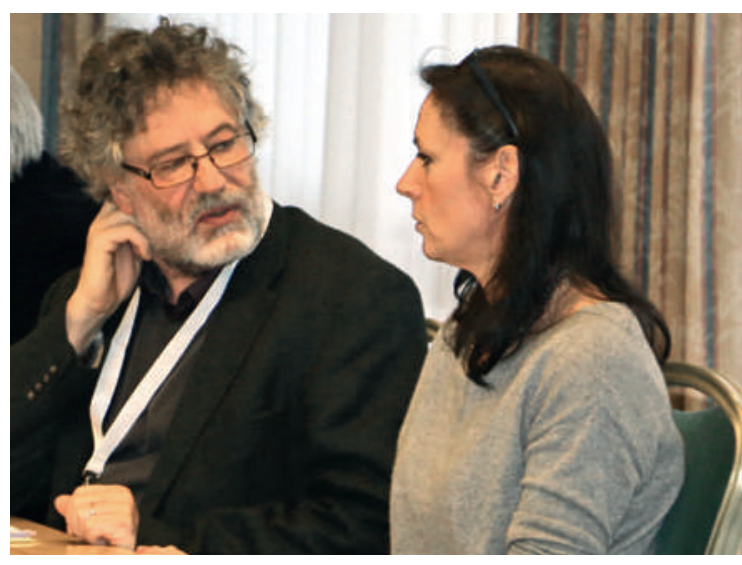

E-Logbuch für Weiterbildner: Peter Eggli tauscht sich mit Iris Baumgartner aus.

keine Handhabe bestehe, von Weiterbildnern ein Mindestmass an Lehrtätigkeit zu verlangen. Zudem hätten diese auch keine Verpflichtung zum Besuch von Fortbildungsveranstaltungen im Sinne von «Teach the Teachers». Als mögliche Lösung regte er die Einführung eines E-Logbuchs für Weiterbildner an, das der Überprüfung eines Mindestmasses von verbindlichen Vorgaben bezüglich Lehrtätigkeit und didaktischer Fortbildung dienen könnte. Der «Samen» für ein Engagement in der Weiterbildung sei idealerweise wohl schon im Studium zu legen. Ein ELogbuch für Weiterbildner schien Stefano Bassetti, Chefarzt Innere Medizin am Universitätsspital Basel, eine zu weit gehende Massnahme, auch wenn er einräumte, dass «ein gewisser Zwang» wohl nicht zu vermeiden sei. Wichtiger sei aber, dass die Weiterbildenden seitens der Universitäten für Ihr Engagement Anerkennung erhielten, was durchaus auch finanziell gemeint war.

Interessante Quervergleiche zur Situation in Deutschland ermöglichte Pascal Berberat, wissenschaftlicher Leiter des Medizin-Didaktischen Centrums für Aus-

\section{«Das Engagement für die Weiterbildung ist} schon da, aber wir haben ein Finanzierungsproblem.»

bildungsforschung und Lehre der Technischen Universität München. Als Arzt in der Schweiz aus- und weitergebildet, kennt der Berner das schweizerische System ebenso wie das deutsche. Er wies darauf hin, dass in Deutschland ein Mindestmass an Lehr- und Ausbildungstätigkeit Voraussetzung für eine Habilitation sei. Die deutschen Erfahrungen zeigten überdies klar, dass Geld halt auch in diesem Bereich eine zentrale Rolle spiele. Das Bonmot dazu lieferte am 


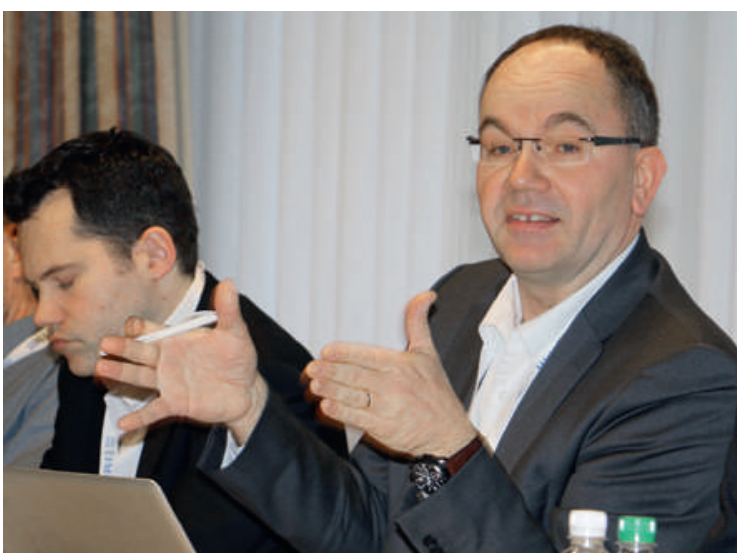

Gute Weiterbildung, schlechte Weiterbildung: Christian Schirlo.

zweiten Tag Christian Schirlo, Stabsleiter der Medizinischen Fakultät der Universität Zürich nach: «Gute Weiterbildung kostet, schlechte noch mehr.»

Dass sich die Diskussion immer wieder um die Finanzierung der ärztlichen Weiterbildung und somit ums Geld drehte, überraschte nicht. Susanna Walitza, ärztliche Direktorin des Kinder- und Jugendpsychiatrischen Dienstes des Kantons Zürich, wies auf den «Gap» zwischen dem pro Assistentenstelle und Jahr ausbezahlten Betrag von 10000 bis 15000 Franken und den tatsächlichen Kosten hin. Diese veranschlagte sie auf rund 50000 Franken pro Jahr. Bernard Vermeulen, Medizinischer Direktor und stellvertretender Generaldirektor des Hôpital de Fribourg bestätigte, dass die ökonomische Realität der Spitäler sehr schwierig sei. «Das Engagement für die Weiterbildung ist schon da, aber wir haben ein Finanzierungsproblem.» Christoph Hänggeli, Geschäftsführer des SIWF, rekapitulierte in diesem Zusammenhang kurz das Modell PEP, auf dem das aktuelle Finanzierungsmodell der Kantone basiert.**

Die Expertinnen und Experten waren sich darin einig, dass Stellenwert und Qualität der Weiterbildung nur durch die von Klaus Grätz angesprochene bessere Vernetzung verbessert werden können. Einige bereits existierende gute Beispiele dafür wurden am Symposium genannt. So berichtete Susanne Walitza, dass im «kleinen Fach» Kinder- und Jugendpsychiatrie bereits ein Verbund bestehe, der sich um die Verbesserung der Weiterbildungsqualität kümmere. Pierre-André Michaud von der Universität Lausanne, dem Werner Bauer ad hoc den Titel «Papst der Ausbildungsziele» verlieh, wies auf die Ecole de formation postgraduée des CHUV und der Universität Lausanne hin, die Ärztinnen und Ärzten bei der Planung der Weiterbildung unterstützt. Hintergrund der spontanen Titelverleihung war Michauds Funktion als

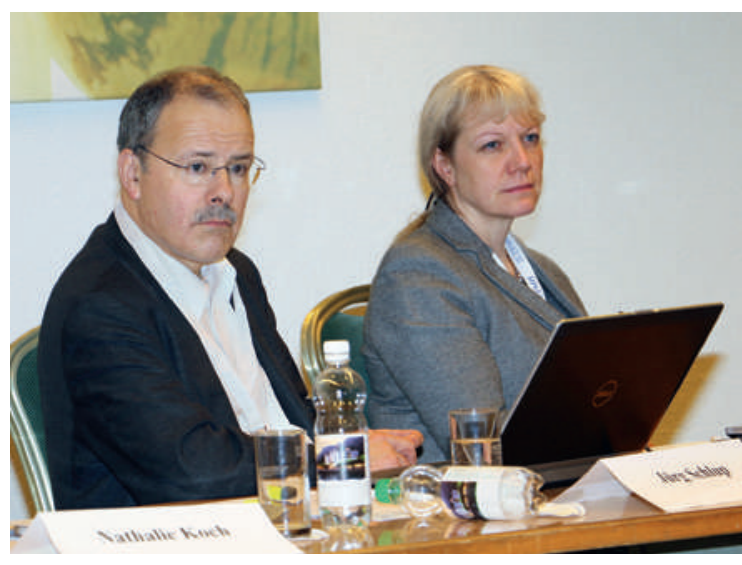

Niedergelassene als Ressource für die Weiterbildung: Jürg Schlup und Susanna Walitza.

Leiter der Kommission zur Revision des SCLO, des Lernzielkatalogs für das Studium. Die Bildung von Spitalnetzwerken, in denen die Assistenzärztinnen und -ärzte im Hinblick auf ihr Weiterbildungsziel ein strukturiertes Curriculum durchlaufen können, ist aus seiner Sicht ein zukunftsträchtiges Modell und ein wichtiges Element der medizinischen Nachwuchsförderung. Auch die Haus- und niedergelassene Fachärzte, so Michaud, sollten vermehrt in die Aus- und Weiterbildungscurricula integriert werden. Ein Ball, den FMH-Präsident Jürg Schlup aufgriff. Erfahrene Praktiker seien eine Ressource, die für die Weiterbildung junger Ärztinnen und Ärzte ohne Zweifel noch besser genutzt werden könnte und sollte, meinte er, und verwies auf einige erfolgreiche Programme in der Deutschschweiz.

Für Peter Meier-Abt, Präsident der Schweizerischen Akademie der Medizinischen Wissenschaften SAMW, könnte das SIWF bei der angestrebten Netzwerkbildung eine Schlüsselposition einnehmen. Regionale Konkurrenz stehe einer an sich sinnvollen Annäherung zunächst häufig im Weg. Das SIWF als neutrale Instanz könne Hindernisse aus dem Weg räumen und Anstösse zu Zusammenarbeit und Verbundbildung geben. Sinnvoll fände er auch eine Vertretung des SIWF durch Delegierte in den Universitätsspitälern. Dadurch könnte der Stellenwert der Weiterbildung in diesen Spitälern unterstrichen und hochgehalten und allfälligen Diskussionen, Weiterbildungsstellen zugunsten von Facharztstellen abzubauen, begegnet werden.

\section{Steuerung der ärztlichen Weiterbildung: ein schwieriges Terrain}

Einen Themenschwerpunkt des zweiten Halbtags bildete die Frage der Steuerung der ärztlichen Weiter- 


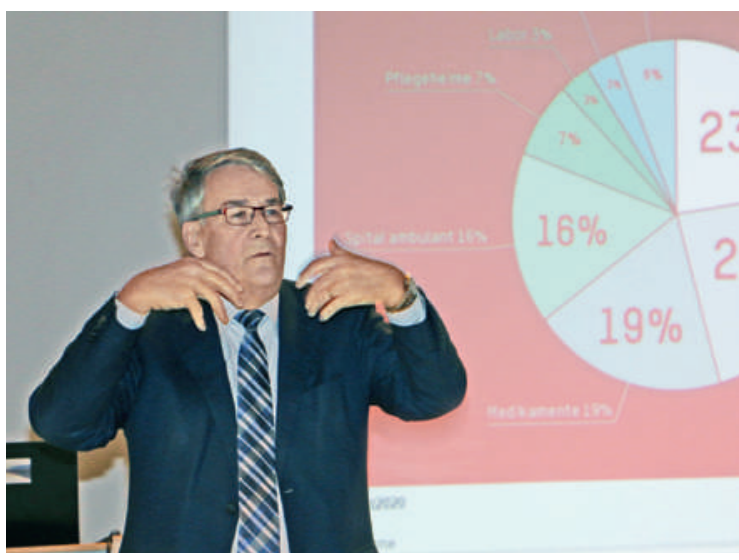

«Es braucht einen Piloten»: Ständerat Urs Schwaller bei seinem Referat.

bildung. Ständerat Urs Schwaller, der für einen «Gedankenaustausch" an die Tagung eingeladen worden war, hatte die Diskussion darüber in seinem Kurzreferat am Vortag bereits lanciert. Auch wenn er sich grundsätzlich als klaren Befürworter des Föderalismus positionierte, ortete er im Gesundheitsbereich "eher zu viel davon». Er räumte ein, dass im Parlament wenig über Weiterbildung und Qualität diskutiert werde, vermutete aber, dass auch in diesem Bereich eine gewisse Steuerung sinnvoll wäre. Die Frage, wie "dirigistisch» man sein wolle, sei indes schwierig zu beantworten. Auf den Hinweis, es gebe

Klar ist für Werner Bauer, «dass wir kein regulatorisch-planwirtschaftliches System wollen".

Bedenken, der Bund beziehungsweise das BAG könnten bei der Steuerung der Weiterbildung zu viel Macht erhalten, antwortete er mit der Frage, ob man sich denn einen anderen Steuermann vorstellen könne, beispielsweise die Krankenkassen. Dem hielt Jürg Schlup entgegen, die Lösung liege nicht in «mehr Staat», das heisst in verstärkter staatlicher Steuerung, sondern in einem Gesundheitsgesetz, das es bisher nicht gebe, im Gegensatz etwa zum Landwirtschaftsgesetz.

Hans Hoppeler, Vizepräsident der Medizinalberufekommission (MEBEKO), fasste den allgemeinen Tenor treffend mit der Feststellung zusammen, ein derart komplexes System könne ohne ein gewisses Mass an zentraler Führung wohl nicht funktionieren. Darüber, wie eine sinnvolle Steuerung im Bereich der ärztlichen Weiterbildung gestaltet werden könnte, wird auf der von BAG und GDK initiierten, breit abgestützten «Plattform ärztliche Bildung» nachgedacht und diskutiert, ohne dass das Ei des Ko-

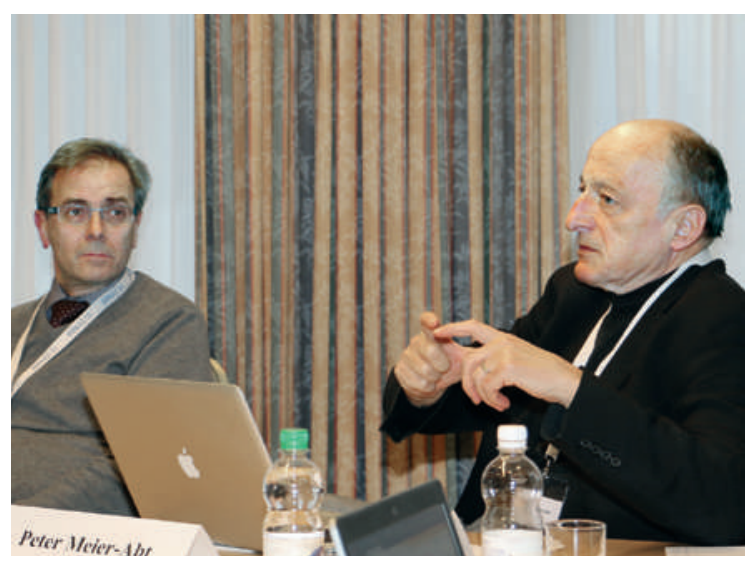

Peter Meier-Abt (rechts) plädierte für eine Führungsrolle des SIWF bei der Netzwerkbildung (links im Bild Bernard Vermeulen).

lumbus bisher gefunden worden wäre. Klar ist für Werner Bauer, «dass wir kein regulatorisch-planwirtschaftliches System wollen", und dass ein solches System auch vom BAG nicht angestrebt wird. Auch in diesem Zusammenhang tauchte verschiedentlich das Zauberwort «Incentives» auf. Für Jürg Hodler, Ärztlicher Direktor des Universitätsspitals Zürich, sind viele der diskutierten und in Studien wie derjenigen des Büro Bass*** vorgestellten Steuerungsmechanismen «rote Tücher», weil sie vom Markt umgangen werden könnten.

Nicht weniger lebhaft, wenn auch zeitlich gedrängter, waren die Diskussionen zu den Themen «Lernziele» (Hintergrund: Revision des schweizerischen Lernzielkatalogs für das Medizinstudium), neue Lehrund Lernmethoden, medizinische Informatik und MOC (Maintenance of Certification) - an Stoff für Reflexionen mangelte es an diesem Symposium wahrlich nicht.

\section{Die europäische Perspektive}

Gastreferent Romuald Krajewski war dafür besorgt, dass der Blick der Symposiumsgäste über Alpengipfel und Jurahöhen hinausschweifte und sich der europäischen Weiterbildungslandschaft zuwandte. Als Präsident der Union Européenne des Médecins Spécialistes UEMS kennt der Warschauer Professor für Neurochirurgie sowohl die allgemeinen Trends als

«Bureaucracy may help reporting, but it will not help patients.»

auch die spezifischen Probleme einzelner Länder und Fachdisziplinen bestens. «Normalerweise kommt man in die Schweiz, um zu lernen, was man alles besser machen kann - aber offenbar habt ihr hier auch 


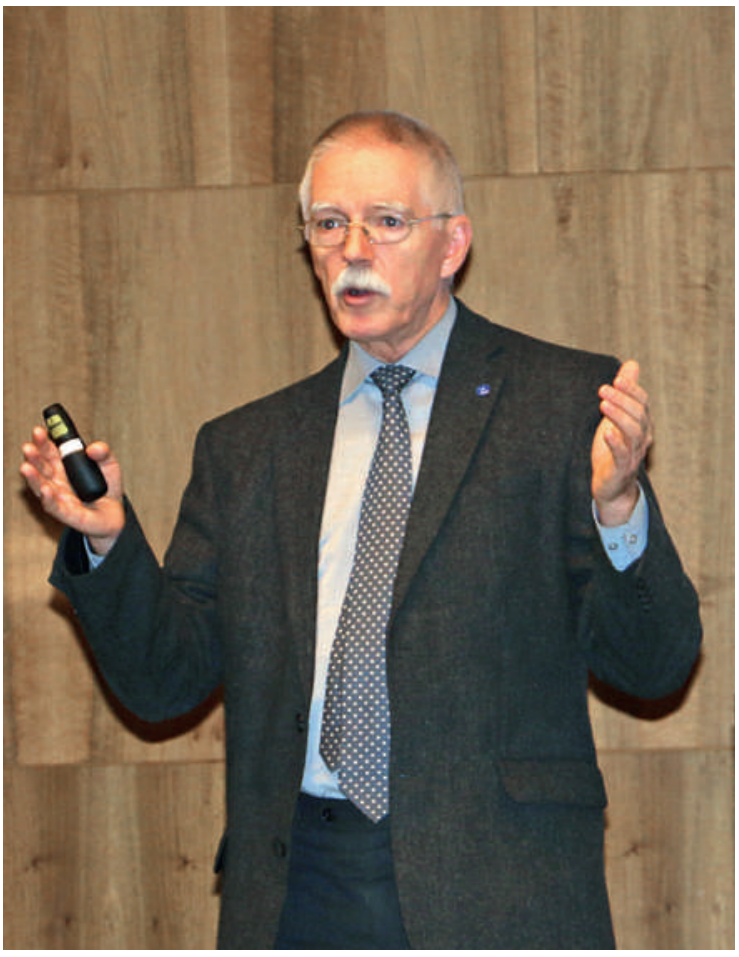

"Offenbar habt ihr hier auch ein paar Probleme»: Romuald Krajewski, Präsident der UEMS.

ein paar Probleme», eröffnete er seinen Vortrag und erntete damit gleich ein paar Lacher. Dass er seinen Humor als UEMS-Präsident nicht verloren hat, scheint angesichts der Aufgaben, denen er sich gegenübersieht, nicht selbstverständlich. Einiger sarkastischer Bemerkungen über die auf europäischer Ebene wuchernde Bürokratie konnte er sich allerdings nicht enthalten. «Bureaucracy may help reporting, but it will not help patients», lautete eine seiner Erkenntnisse. Insbesondere im Bereich der Qualitäts- sicherung stelle er eine Vielzahl rein politisch motivierter Vorstösse fest, die absolut nichts brächten.

Die UEMS hat sich unter anderem zum Ziel gesetzt, europaweit einheitliche Standards bei der Facharztausbildung zu schaffen. Die Finanzierung dieser Vereinheitlichung ist aber offenbar so schwierig, dass Krajewski das Projekt vorsichtig als "Traum» bezeichnete. $\mathrm{Zu}$ den Schlüsselaktivitäten zählen auch Anstrengungen für Verbesserungen im Bereich der Fortbildung (Continuing medical education CME, Continuing professional development CPD) und der Behandlungsqualität. Letztere soll in Europa flächendeckend auf ein gutes Niveau gebracht werden. Die UEMS setzt sich für eine hohe Autonomie des ärztlichen Berufsstandes ein und hat Selbstregulation, Mobilität in der Aus- und Weiterbildung sowie der Berufsausübung, interdisziplinäre Zusammenarbeit und Teamwork als weitere "Core values" definiert. Die Diskussion über die technischen Möglichkeiten in der Weiter- und Fortbildung, aber auch bei der Behandlung von Patienten ist auch auf europäischer Ebene in vollem Gang. Krajewski zeigte sich überzeugt, dass Online- und virtuelle Ausbildungsmöglichkeiten "Live events» auch in Zukunft nicht vollständig ersetzen könnten und dass die Patienten ihre Ärztinnen und Ärzte in Zukunft vermehrt wieder «als Menschen» und weniger als «Techniker» brauchen würden.

Ein Gedanke, an den Klaus Grätz in seinem Schlusswort anknüpfte, als er den Stellenwert des Vertrauens in der ärztlichen Tätigkeit betonte und in Erinnerung rief, dass die optimale Versorgung der Patienten auch in ökonomisch schwierigen Zeiten die ärztliche Hauptaufgabe bleibe. Dass daran - zu Recht - erinnert wurde, sagt auch einiges über die Zeit aus, in der wir leben. 